\title{
Effects of strontium ranelate on bone formation in the mid-palatal suture after rapid maxillary expansion
}

\author{
Shuya Zhao',* \\ Xuxia Wang ${ }^{2, *}$ \\ $\mathrm{Na} \mathrm{Li}^{3}$ \\ Yun Chen' \\ Yuran Su' \\ Jun Zhang' \\ 'Department of Orthodontics, \\ ${ }^{2}$ Department of Oral and \\ Maxillofacial Surgery, Faculty of \\ Stomatology, Shandong University; \\ ${ }^{3}$ Department of Orthodontics, \\ Shandong Provincial Qianfoshan \\ Hospital, Jinan, People's Republic \\ of China \\ *These authors contributed equally \\ to this work
}

This article was published in the following Dove Press journal:

Drug Design, Development and Therapy

21 May 2015

Number of times this article has been viewed

Background: The aim of this experimental study was to investigate the effects of strontium ranelate on bone regeneration in the mid-palatal suture in response to rapid maxillary expansion (RME).

Methods: Thirty-six male 6-week-old Wistar rats were randomly divided into three groups, ie, an expansion only (EO) group, an expansion plus strontium ranelate (SE) group, and a control group. An orthodontic appliance was set between the right and left upper molars of rats with an initial expansive force of $0.98 \mathrm{~N}$. Rats in the SE group were administered strontium ranelate (600 mg/kg body weight) and then euthanized in batches on days 4, 7, and 10. Morphological changes in the mid-palatal suture were investigated using micro-computed tomography and hematoxylin and eosin staining after RME. Bone morphogenetic protein-2 expression in the suture was also examined to evaluate bone formation in the mid-palatal suture. Image-Pro Plus software was then used to determine the mean optical density of the immunohistochemical images. Analysis of variance was used for statistical evaluation at the $P<0.05$ level.

Results: With expansive force, the mid-palatal suture was expanded, but there was no statistically significant difference $(P>0.05)$ between the SE and EO groups. The bone volume of the suture decreased after RME, but was higher in the SE group than in the EO group on days 7 and 10. Further, expression of bone morphogenetic protein-2 in the SE group was higher than in the other two groups $(P<0.05)$.

Conclusion: Strontium ranelate may hasten new bone formation in the expanded mid-palatal suture, which may be therapeutically beneficial in prevention of relapse and shortening the retention period after RME.

Keywords: strontium ranelate, rapid maxillary expansion, micro-computed tomography, bone morphogenetic protein-2, bone formation

\section{Introduction}

Clinically, rapid maxillary expansion (RME) is widely used for growing children in the correction of malocclusions, such as crowding, arch stenosis, and posterior cross-bite. The mechanical strains of the expansion appliance can be conducted at the mid-palatal suture through the molars and bone, then expand the posterior dentition width rapidly and trigger a series of biologic activities to accelerate new bone formation in the midpalatal suture. ${ }^{1-3}$ The suture tissues go through bone formation, resorption, and fiber rearrangement until a new equilibrium is reached. In this process, however, several undesirable results are observed, such as external root resorption, ${ }^{4}$ microtrauma of the temporomandibular joint, ${ }^{5}$ and post-expansion relapse.$^{6}$ The relapse phenomenon after RME is one of the most frequently encountered complications, and the incidence
Correspondence: Jun Zhang

Department of Orthodontics, Stomatological Hospital of Shandong University, Shandong University, 44

Wenhua West Road, Lixia District, Jinan 2500 I2, People's Republic of China

Tel +86053 I 88382070

Fax +86 053। 88382070

Email zhangj@sdu.edu.cn 
may reach as high as $45 \% .^{5,7-9}$ Further, surgically assisted $\mathrm{RME}$ is feasible for non-growing adults or skeletally mature patients. Although the rigid internal fixation technique is used in some surgically assisted RME procedures to fix the bone and prevent contraction, orthodontic treatment and a fixed retainer are recommended by many investigators to achieve sufficient retention and stability. ${ }^{10-15}$ Therefore, there is no good method to prevent the post treatment relapse in orthodontics and surgery. The expanded suture tends to return to the pretreatment width. Many researchers argue that one of the major causes of early relapse after expansion could be insufficient bone regeneration in the mid-palatal suture, and have tried certain methods to improve regeneration capacity in the mid-palatal suture, including laser therapy ${ }^{1,16}$ and agents such as lithium chloride, ${ }^{2}$ vitamins, ${ }^{6,17}$ bisphosphonates, ${ }^{18}$ dietary boron, ${ }^{19}$ and antioxidants. ${ }^{20,21}$ Promoting new bone formation and mineralization in the mid-palatal suture after RME will be helpful to resist the retractive force produced by surrounding tissue components and to maintain the stability of the maxilla structure, significantly shorten the retention period after expansion, and prevent relapse of the arch width, which would popularize expansion treatment in orthodontic patients and bring some new perspectives to combined surgical and orthodontic treatment.

Strontium ranelate (SrR), a divalent strontium salt of ranelic acid, is a new pharmacologic agent for osteoporosis. A number of experiments have demonstrated that SrR can not only prevent bone resorption but can also promote bone formation. $^{22-24}$ Considering its stimulatory effects on bone formation, the aim of this study was to evaluate the effects of $\mathrm{SrR}$ on bone regeneration in response to expansion of the mid-palatal suture in rats. In this study, we hope to provide new additional insights into strategies for preventing postexpansion relapse.

\section{Materials and methods}

\section{Animals and groups}

For this study, we obtained 36 male Wistar rats (6 weeks old, mean weight $150 \pm 10 \mathrm{~g}$ ) from the experimental animal center at Shandong University, Jinan, People's Republic of China. The rats were kept separately in plastic cages under artificial lighting by fluorescence lamps with a 12:12-hour light:dark cycle. The temperature of the cages was set at $25^{\circ} \mathrm{C}$, and the rats were fed on a normal rodent diet with a minimum of $0.1 \%$ calcium content, $0.4 \%$ phosphorus content, and 2,000 IU/kg vitamin D content. Fresh drinking water was provided every day, and the interior noise was controlled below $60 \mathrm{~dB}$. The body weight and health of all rats were checked daily.
The study was approved by the Institutional Animal Care and Use Committee of Shandong University, and was carried out in accordance with the National Institutes of Health Guidelines for the Use of Laboratory Animals.

The rats were randomly divided into three groups $(n=12$ each): an expansion only (EO) group, an expansion plus SrR (SE, where SrR was given to the rats during the expansion period) group, and a control group (where the rats received no procedure or $\mathrm{SrR}$ ).

\section{Mid-palatal suture expansion procedure}

The rats in the EO and SE groups were anesthetized by an intraperitoneal injection of $2 \%$ ketamine hydrochloride $2 \mathrm{~mL} / \mathrm{kg}$ (Jiangsu Hengrui Medicine Co Ltd, Lianyungang, People's Republic of China) during setting and adjusting of the orthodontic appliance. Once anesthetized, the rats were kept supine on the operating table, with the extremities and head fixed. The maxillary modulus of the rats was taken using a homemade tray to achieve an individual impression and the working model. According to the model, a stainless steel orthodontic wire (0.014 inch heat-treated arch wire, AJ Wilcock Pty Ltd, Whittlesea, Australia) was bent into a rectangular form with two opening loops (Figure 1A). After the appliances were bonded to the first and second maxillary molars on both sides, light-cured adhesive (Gluma Comfort Bond, Heraeus Kulzer GmbH, Hanau, Germany) was attached to the molars of the rats after etching the teeth with acid etch (Gluma Etch 35 Gel, Heraeus Kulzer GmbH). The initial expansive force was adjusted to $0.98 \mathrm{~N}$, measured with a strain gauge (Hangzhou Aosu Medical Device Co, Hangzhou, People's Republic of China). The occlusal view of the orthodontic appliance in the maxilla of the rat is shown in Figure 1B. During surgery, the rats were kept warm by lighting in order to maintain body temperature, and vital signs were closely observed until the rats were fully awake.

\section{Administration of solution}

SrR for suspension obtained from Servier Pharmaceutical R\&D Co Ltd (Tianjing, People's Republic of China), were dissolved in water. In the SE group, SrR $600 \mathrm{mg} / \mathrm{kg}$ body weight was administered daily via the orogastric route. Dosing was performed at the same time each day until the rats were euthanized.

\section{Observation and measurement of weight}

All animals survived to the end of the experiment. Body weight was measured every day throughout the study period. The animals were also monitored for infection or appliance 
A

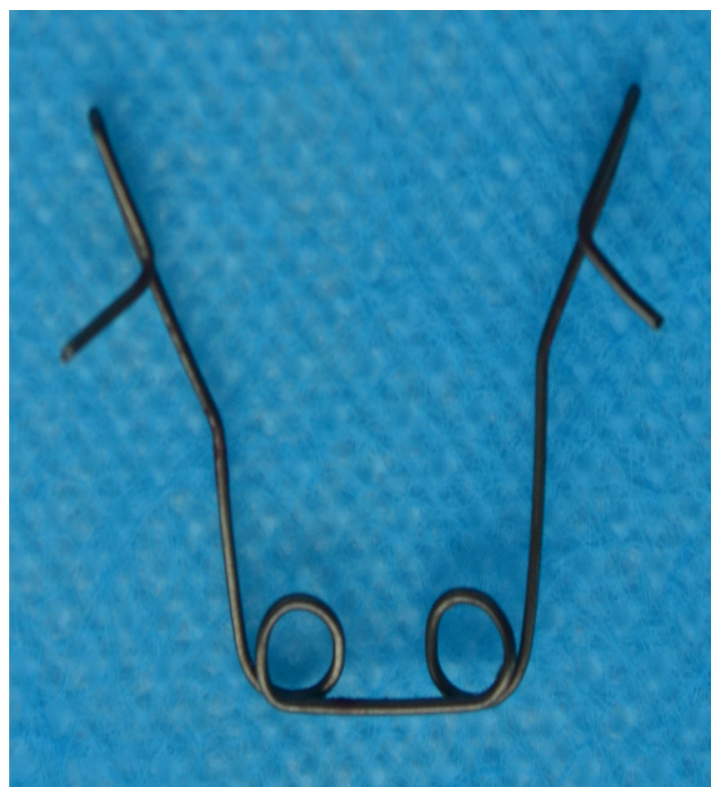

B

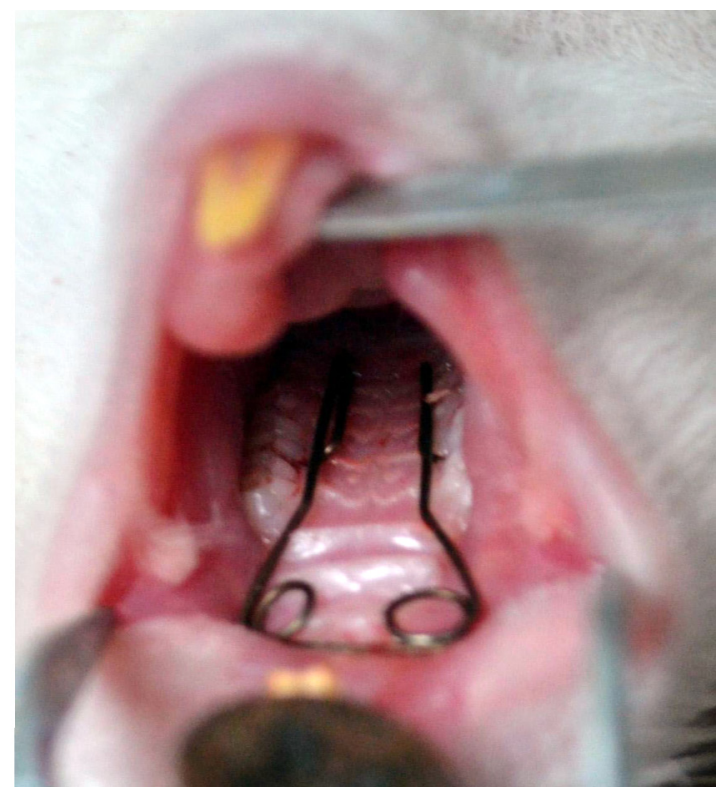

Figure I Orthodontic mechanics used in this study.

Notes: (A) The appliance with the expansive force. The initial force was adjusted to $0.98 \mathrm{~N}$. (B) Intraoral view of orthodontic appliance set on the rat.

failure throughout the study. If any complication, such as mucosal infection, a rapid decrease in body weight, or a detached appliance, was observed, the animal was excluded from the study.

\section{Specimen preparation}

The rats were euthanized in batches on days 4,7 , and 10 by injecting an overdose of ketamine hydrochloride after orthodontic treatment. Four animals in each group were sacrificed in each batch. The rat maxillae were dissected and fixed in $4 \%$ paraformaldehyde buffer at $4{ }^{\circ} \mathrm{C}$ for 24 hours. The maxillary bone was surgically removed, trimmed, and decalcified in $10 \%$ ethylenediaminetetraacetic acid/phosphate-buffered saline solution at $4^{\circ} \mathrm{C}$ for 3 months. During decalcification, the solution was replaced once every 3 days. Next, the samples were subjected to graded ethanol dehydration and cleared with xylene, then embedded in paraffin wax for tissue sectioning.

\section{MicroCT analyses of the hard palate}

Before decalcification, the whole maxilla was scanned using a micro-computed tomography (CT) system (Inveon MM, Siemens, Knoxville, TN, USA), after which threedimensional and two-dimensional radiographs of the hard palate were obtained. The specimens were scanned at $80 \mathrm{kV}, 500 \mu \mathrm{A}$, and $8.5 \mu \mathrm{m}$ effective pixel size. The images were analyzed using Inveon Research Workplace software (Siemens). COBRA reconstruction software (Exxim
Computing Corporation, Pleasanton, CA, USA) was used to construct three-dimensional images to observe morphological changes in the mid-palatal suture (Figure 2A and B). The width of the mid-palatal suture was measured at the level of the mid-coronal plane of the upper first molar (Figure 2C). Meanwhile, the region of interest (ROI) within which bone volume was measured $(0.60 \mathrm{~mm} \times 0.90 \mathrm{~mm} \times 2.00 \mathrm{~mm})$ consisted of the mid-palatal suture and the bilateral bones (Figure 2D-F). The area in the blue box is a target area, while the area in the green box represents bone. The bone volume change in the ROI was obtained to investigate the effects of expansive force and SrR on the mid-palatal suture.

\section{HE staining}

Serial frontal $5 \mu \mathrm{m}$ thick sections of the embedded tissues were cut at the level of the upper first molar using a slicer (EG115OH, Leica, Wetzlar, Germany) and mounted on slides coated with 3-aminopropyl-triethoxysilane. After the sections were deparaffinized and rehydrated, they were prepared for hematoxylin and eosin (H\&E) and subsequent immunohistochemical staining. For histological examination, the sections were stained with $\mathrm{H} \& \mathrm{E}$ and observed under a light microscope (BX51, Olympus, Tokyo, Japan). The histological changes and bone remodeling are shown in Figure 3.

\section{Immunohistochemical staining of BMP-2}

The sections were deparaffinized in xylene, hydrated through a graded alcohol series, and washed with phosphate-buffered 

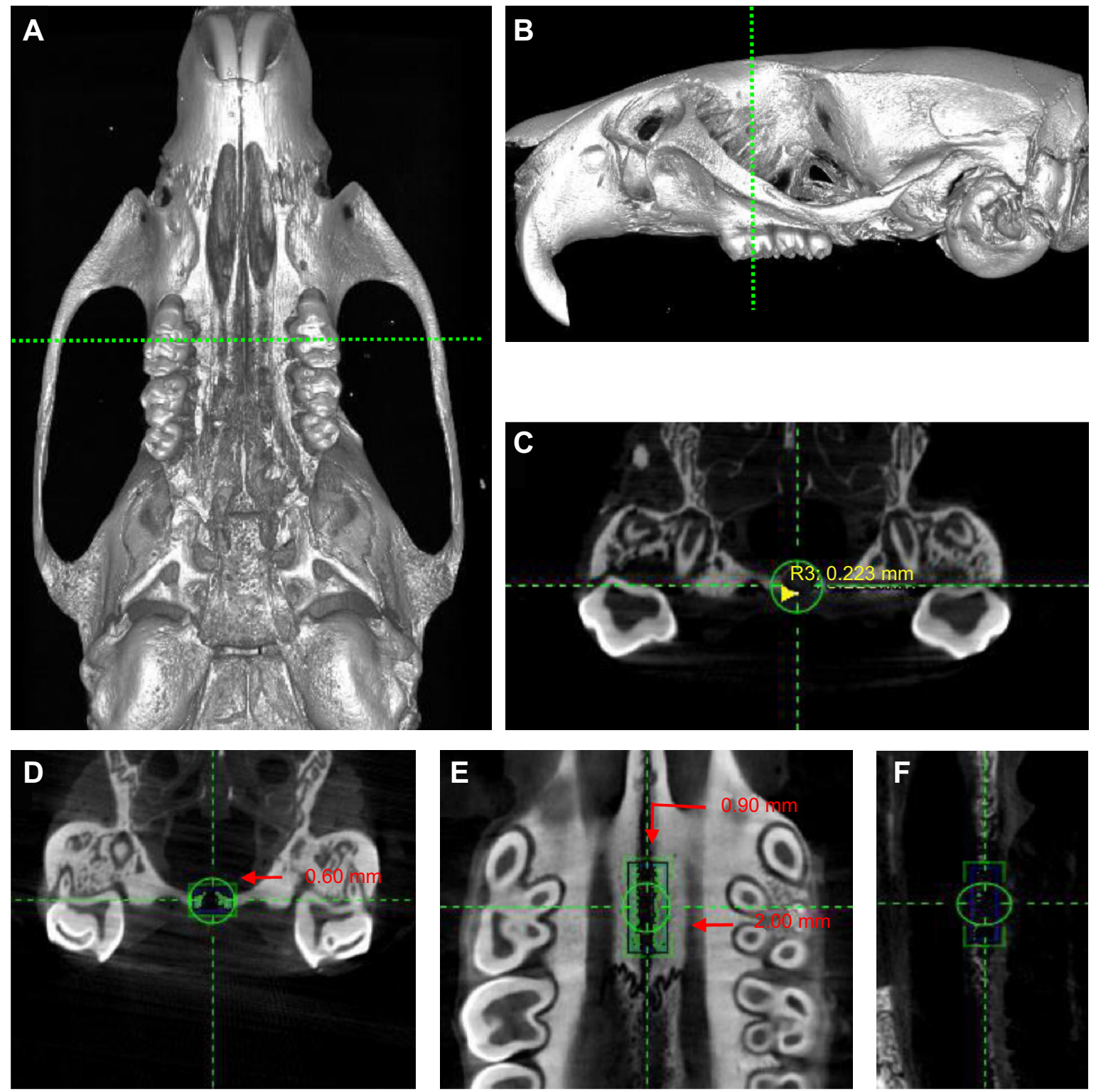

Figure 2 Three-dimensional image of the craniomaxillary tissue of the rat and diagram of the region of interest $(\mathrm{RO} ; 0.60 \mathrm{~mm} \times 0.90 \mathrm{~mm} \times 2.00 \mathrm{~mm})$.

Notes: The rat maxilla was dissected and scanned by microCT to observe the morphological changes in the mid-palatal suture. The bone volume was measured within the $\mathrm{ROI}$ (the area in the blue box represented ROI, and the area in the green box marked the bone volume). (A) MicroCT image displaying the occlusal view of the rat head with a green dotted line marking the occlusal position of the mid-coronal plane of the upper first molar. (B) MicroCT image displaying the sagittal view of the rat head with a green dotted line marking the sagittal position of the mid-coronal plane of the upper first molar. (C) MicroCT image displaying the mid-coronal plane where the mid-palatal width was measured. (D) The coronal plane of the rat head. (E) The horizontal plane of the rat head. (F) The sagittal plane of the rat head.

Abbreviations: ROI, region of interest; microCT, micro-computed tomography.

saline. Antigen retrieval was performed by treatment with $0.1 \%$ (w/v) trypsin (Zhongshan, Beijing, People's Republic of China) at $37^{\circ} \mathrm{C}$ for 10 minutes. The activity of endogenous tissue peroxidase was blocked with $3 \% \mathrm{H}_{2} \mathrm{O}_{2}$ (Zhongshan) for 30 minutes. After pretreatment with normal goat serum (Zhongshan) for 30 minutes to block nonspecific binding, the sections were incubated with bone morphogenetic protein (BMP)-2 antibody (1:100 dilution; Biosythesis Biotechnology, Beijing, People's Republic of China) at $4^{\circ} \mathrm{C}$ overnight. The sections were then incubated with biotinylated goat antirabbit immunoglobulin $\mathrm{G}$ and streptavidin-biotin complex
(Boster, Wuhan, People's Republic of China) at $37^{\circ} \mathrm{C}$ for 25 minutes. Diaminobenzidine solution (Boster) was used to visualize localization for 2 minutes. Finally, the sections were lightly counterstained with hematoxylin. Sections treated with phosphate-buffered saline instead of the primary antibody were used as negative controls.

Cells with brownish-yellow granules on the cytoplasm or nucleus were deemed to be positive cells. The slides were analyzed by Image-Pro Plus software to determine the mean optical density of the immunohistochemical images. A single examiner in a blind study randomly selected five high power 

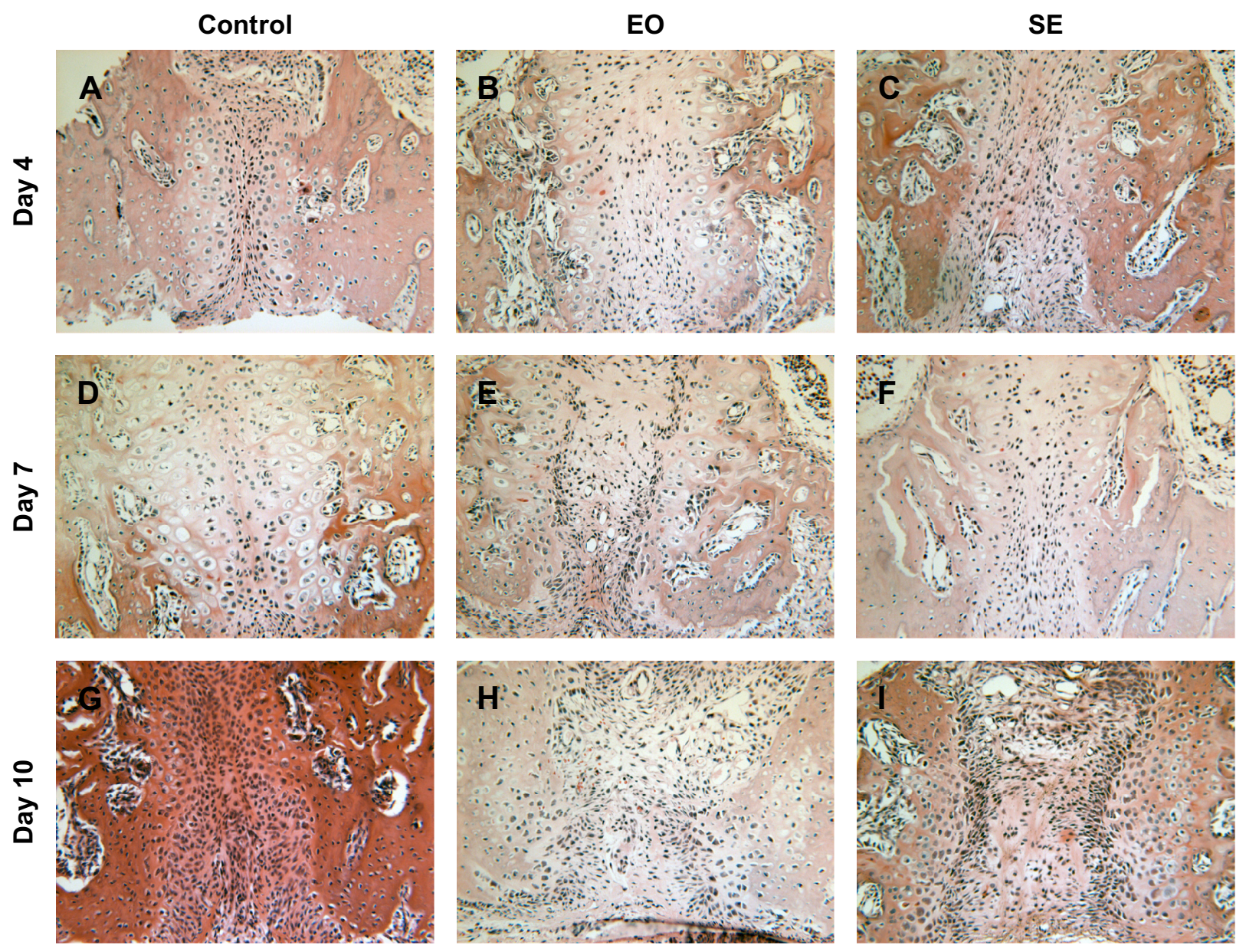

Figure 3 Hematoxylin and eosin staining of frontal section of the mid-palatal suture in 6-week-old rats (all images are displayed at $200 \times$ magnification and oriented with the nasal side up and the oral side down).

Notes: (A-C) The mid-palatal suture of the control, EO, and SE groups on day 4. (D-F) The mid-palatal suture of the control, EO, and SE groups on day 7. (G-I) The midpalatal suture of the control, EO, and SE groups on day 10.

Abbreviations: EO, expansion only group; $\mathrm{SE}$, expansion plus strontium ranelate group.

fields (400×) from each slide to carry out the evaluation and the results were taken as an average of five fields.

\section{Statistical analysis}

All data are presented as the mean \pm standard deviation for each group and analyzed using Statistical Package for the Social Sciences version 19.0 (SPSS Inc, Chicago, IL, USA). Kruskal-Wallis one-way analysis of variance was used to compare the groups. To identify intergroup differences, we used the least square difference method. Probability values $<0.05$ were considered to be statistically significant.

\section{Results}

\section{Body weight changes}

The animals with the expansion application did not suffer from mucosal infection, dehiscence, or other adverse effects throughout the study period and there were no rodent deaths. The body weight of the rats in the control group increased steadily. The body weight of rats in the EO and SE groups decreased on days 2 and 3, but subsequently recovered on day 4 . On the first 2 days of rapid mid-palatal expansion, the rats with the expansion appliance had difficulty in eating and were uncomfortable. However, there was no evidence of diarrhea or other gastrointestinal symptoms in any of the animals; therefore, body weight slightly decreased in the SE and EO groups at the beginning of expansion and then recovered. There were significant differences in mean body weight between the control group and the other two groups from day 2 to day $7(P<0.05)$, but there were no significant differences between the $\mathrm{SE}$ and $\mathrm{EO}$ groups at any time during the study ( $P>0.05$, Figure 4$)$.

\section{Morphological changes of the mid-palatal suture by microCT}

The width of the mid-palatal suture was measured from the three-dimensional images of microCT. The mid-palatal suture was expanded by the activated RME application. In the EO and SE groups, a significant increase in the width of the suture $(P<0.05)$ and in the tipped molars was observed on days 4 , 


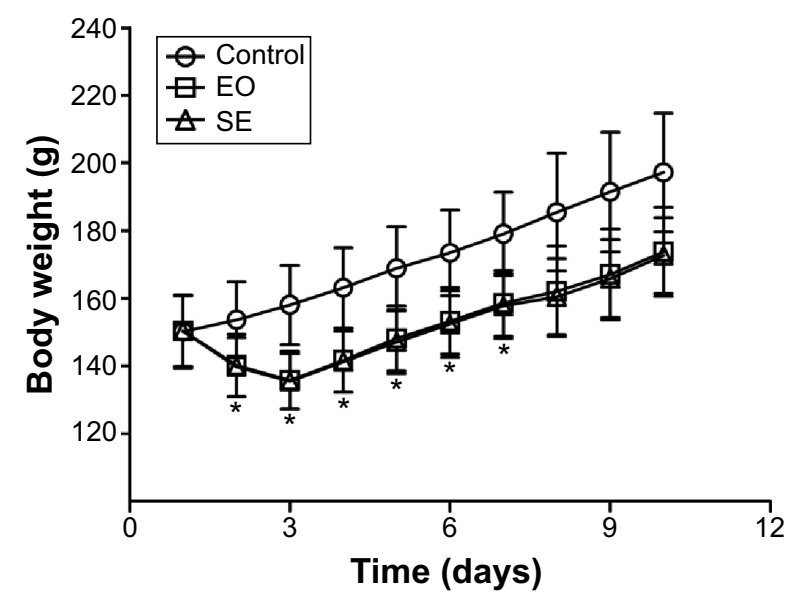

Figure 4 Body weight changes of rats in the three groups during the experimental period.

Notes: $* P<0.05$, significant decrease versus the control group.

Abbreviations: EO, expansion only group; SE, expansion plus strontium ranelate group.

7, and 10. The mean amount of expansion was less in the SE group than in the EO group on days 7 and $10(0.386 \pm 0.013 \mathrm{~mm}$ and $0.391 \pm 0.022 \mathrm{~mm}, 0.370 \pm 0.012 \mathrm{~mm}$ and $0.380 \pm 0.013 \mathrm{~mm}$, respectively). However, the statistical analysis showed no significant differences $(P>0.05$, Figure 5).

Bone formation in the mid-palatal suture after RME was observed by increases and decreases in bone volume at the ROI. There was no obvious change in the control group during the study, but the SE and EO groups had a significant decrease in bone volume in the ROI compared with the control group $(P<0.05)$. The bone volume at the ROI in the SE group was more than that in the EO group on days 7 and 10 $(P<0.05$, Figure 6).

\section{HE staining}

HE staining showed detailed histological changes in the midpalatal suture (Figure 3A-I). The suture is made up of central

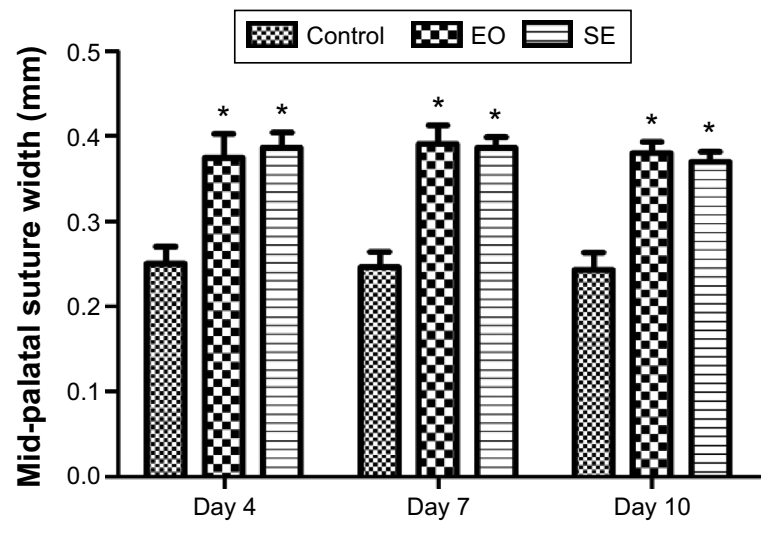

Figure 5 The width of the mid-palatal suture of the three groups. Notes: $* P<0.05$, significant increase versus the control group.

Abbreviations: EO, expansion only group; SE, expansion plus strontium ranelate group.

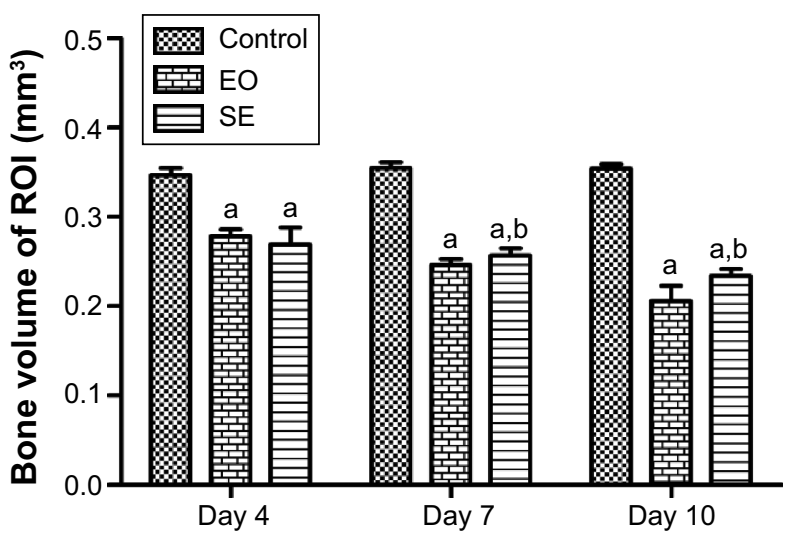

Figure 6 Bone volume change in the ROI.

Notes: a: $P<0.05$, significant decrease versus the control group; b: $P<0.05$, significant increase versus the EO group.

Abbreviations: EO, expansion only group; SE, expansion plus strontium ranelate group; ROI: region of interest.

fibrous tissue and lateral secondary cartilage that is a progression of small-sized cells to mature chondrocytes close to the bony edges. Under the expansive force, the mid-palatal suture was widened and bone formation in the cartilaginous tissue enhanced. In the control group, the mid-palatal suture stayed almost unchanged. There were slight changes in the number of chondrocytes and the amount of fibrous tissue increased, but there was almost no change in the width of the suture throughout the study period. After expansion, the EO and SE groups had expanded cartilaginous masses extending into the fibrous layer. The chondrocytes proliferated, differentiated, became hypertrophic, and some were replaced by osteoblasts that produced bone. Over time, the new bone formation continued and formed columnar bony structures growing in the center of the suture. Bone formation in the SE group was obvious compared with the EO group on days 7 and 10. This histological observation, in accordance with the microCT results, indicated that more deposition of new bone matrix occurred in the mid-palatal suture when stimulated by SrR.

\section{Immunohistological findings for BMP-2}

The immunohistochemical examination is shown in Figure 7. In the control group, very little expression of BMP-2 was seen in the osteogenic zone of the suture. In the EO and SE groups, BMP-2 expression was detected in the osteoblasts, chondrocytes, and endothelial cells inside the blood vessels and fibroblasts in the connective tissue of the suture. Compared with the control group, the mean optical density value of BMP-2 immunoreactivity in the EO group was significantly high $(P<0.05)$ on day 4 but no statistically significant differences were found on days 7 and $10(P>0.05)$. BMP-2 expression in the SE group was more intense $(P<0.05)$ than that of the other two groups during the entire study period (Table 1). 

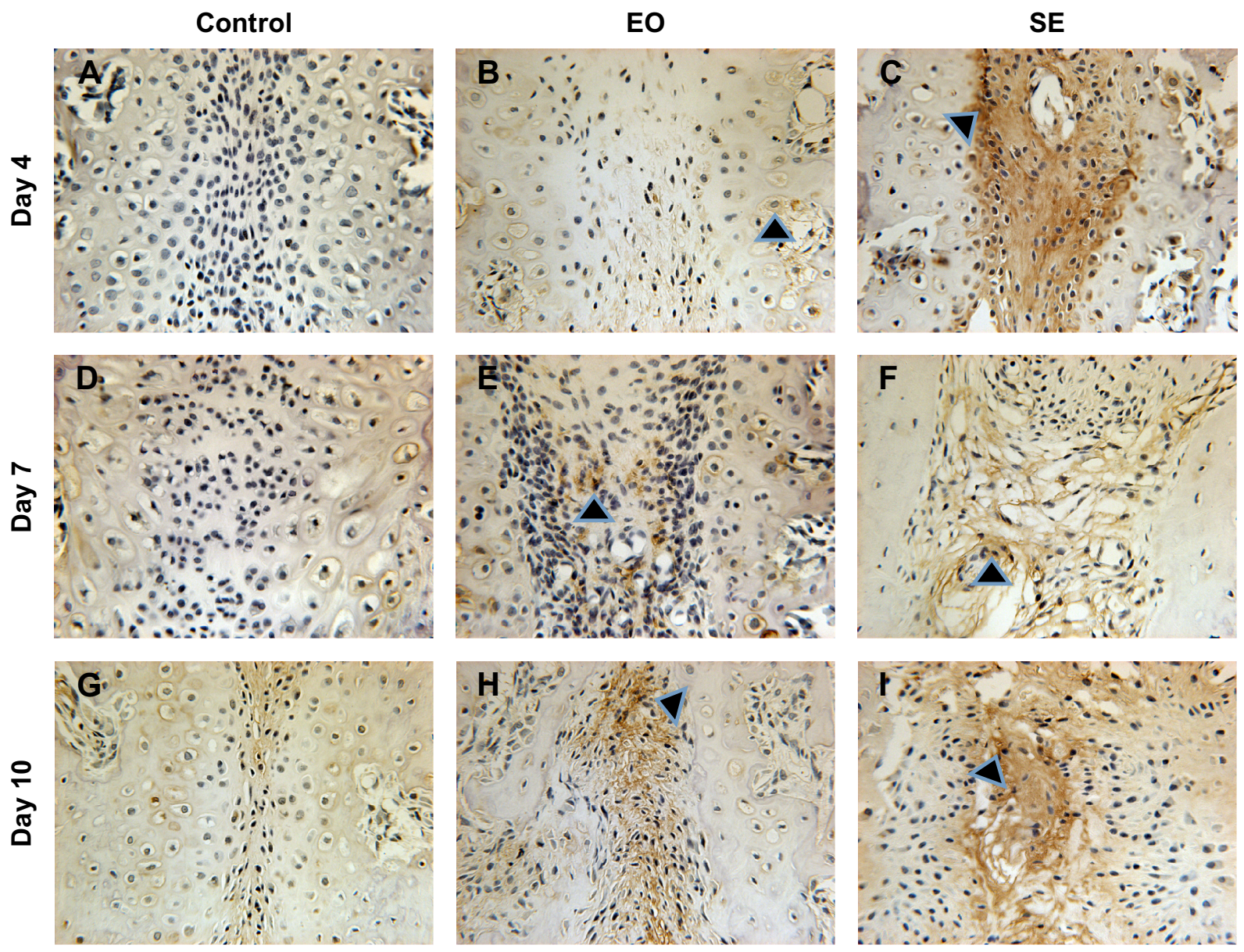

Figure 7 Immunohistochemical staining of BMP-2 (400× magnification).

Notes: Positive cells in the osteogenic zone (arrowhead). (A-C) The mid-palatal suture of the control, EO, and SE groups on day 4. (D-F) The mid-palatal suture of the control, EO, and SE groups on day 7. (G-I) The mid-palatal suture of the control, EO, and SE groups on day 10.

Abbreviations: EO, expansion only group; $\mathrm{SE}$, expansion plus strontium ranelate group; $\mathrm{BMP}$, bone morphogenetic protein.

\section{Discussion}

This study demonstrates that the active effects of SrR on bone formation respond to expansion of the mid-palatal suture in rats. RME is a common method used for correction of transverse maxillary deficiency. ${ }^{25-27}$ However, relapse after RME is a difficult problem that clinicians have to deal with. There are several possible reasons for relapse, including

Table I MOD value of bone morphogenetic protein-2 in the three groups

\begin{tabular}{llll}
\hline Groups & MOD & & \\
\cline { 2 - 4 } & Day 4 & Day 7 & Day 10 \\
\hline Control & $0.014 \pm 0.003$ & $0.015 \pm 0.003$ & $0.019 \pm 0.002$ \\
EO & $0.024 \pm 0.002^{\mathrm{a}}$ & $0.023 \pm 0.005$ & $0.026 \pm 0.004$ \\
SE & $0.038 \pm 0.007^{\mathrm{a}, \mathrm{b}}$ & $0.036 \pm 0.009^{\mathrm{a}, \mathrm{b}}$ & $0.040 \pm 0.008^{\mathrm{a}, \mathrm{b}}$ \\
F-value & 31.787 & 11.797 & 16.930 \\
P-value & 0.000 & 0.003 & 0.001 \\
\hline
\end{tabular}

Notes: Data are presented as mean \pm SD. a $<0.05$ indicates a significant increase versus the control group; ${ }^{\mathrm{b} P}<0.05$ indicates a significant increase versus the $\mathrm{EO}$ group.

Abbreviations: EO, expansion only group; SE, expansion plus strontium ranelate group; SD, standard deviation; MOD, mean optical density. mode of expansion, magnitude of the force, duration of retention, ossification of the mid-palatal suture, and rigidity of the articulation of the zygomatic complex contiguous to the maxilla. ${ }^{6,28,29}$ Further, individual factors, including age, sex, and regulation of bone metabolism, may also influence post-treatment relapse. Although the mechanism for relapse is not fully understood, it would be potentially beneficial to accelerate bone formation in the mid-palatal suture after expansion, shorten the retention period, and prevent relapse of the arch width. Several approaches ${ }^{6,21,30}$ have been investigated for hastening new bone formation to prevent relapse after RME.

SrR is widely used for postmenopausal osteoporosis to reduce the risk of fractures. ${ }^{31,32}$ It has a dual effect on bone regeneration, being able to either promote bone formation or reduce bone resorption. ${ }^{33-35}$ Researchers have documented that $\mathrm{SrR}$ induces osteoblastogenesis by increasing preosteoblast proliferation, osteoblast differentiation, and bone matrix synthesis and mineralization. ${ }^{36,37}$ Additionally, 
SrR can inhibit bone resorption by decreasing osteoclast differentiation and activity. ${ }^{38,39}$ In this study, we found that SrR had potential effects on new bone formation and mineralization in the mid-palatal suture after RME.

The rodent model of maxillary expansion is well established. In the mid-palatal suture of the human maxillary bone, the suture cells and fibers run along the suture parallel to the bone margins. ${ }^{40}$ Although the maxillary sutures of monkeys are more close to those of humans than those of rats, rats are considered to be suitable for animal models to observe bony and sutural changes under stress. ${ }^{1}$ The literature ${ }^{41-43}$ has reported that bone formation reduces with age, that the desired orthopedic effects in the mid-palatal suture can be achieved before and during pubertal growth, and that it is difficult to obtain palatal expansion after the pubertal growth period. Thus, 6-week-old growing rats were used in the present study. In addition, an expansion appliance with a two-coil spring, which is similar to clinical expansion treatment, was placed on the rodent molars. Meanwhile, the initial expansive force of $0.98 \mathrm{~N}$ could expand the midpalatal suture and was within the physiological range of rats because the body weight of the rats subsequently recovered 3 days after expansion.

The mid-palatal suture of growing rats is composed of polygonal mesenchymal cells, mature cartilaginous cells, and osteochondroprogenitor cells that have strong proliferative activity and can differentiate into either chondrocytes or osteoblasts. ${ }^{4-46}$ Histological observation demonstrated that two cartilaginous masses with mature and hypertrophic chondrocytes, commonly referred to as secondary cartilage, were next to each of the bone edges with central fibrous tissue. The increase of the expanded suture width was less apparent on day 10 than on day 7 . This could be due to the gradual reduction of the spring force and the suture gradually reaching a steady state. Expansion of the maxilla mainly results from the extended fibrous tissue in the mid-palatal suture, so SrR cannot decrease the amount of expansion even though it may inhibit the differentiation and activity of osteoclasts. Histology is a good method for analyzing sutures, but microCT can provide three-dimensional anatomic imaging of skeletal structures with efficiency in bone microstructure analysis. ${ }^{47}$ Some investigators ${ }^{48,49}$ have evaluated the structure of the mid-palatal suture using microCT. In this rodent study, we quantitatively evaluated the width change and the bone volume of the ROI in the mid-palatal suture stimulated by expansion and SrR through microCT. We could clearly see the suture was expanded by the expansion application. Current clinical studies have demonstrated that treatment with SrR could increase bone mineral density and bone volume in osteoporotic women. ${ }^{50}$ Our study showed that the maxilla in the control group maintained their bone volume in the mid-palatal suture over 10 days while the SE and EO groups showed significantly decreased bone volume in the ROI. Because the maxilla was expanded, the bone volume in the same-sized ROI should decrease. However, the bone volume in the SE group was more than that in the EO group on days 7 and 10. This demonstrated that SrR could promote formation of new bone, which requires a certain period of time to be observed on CT images. The bone volume measured in the study is in the same-sized area. If more precise measurement is required, the mid-palatal suture should be scanned every day by in vivo microCT. Unfortunately, the radiation the living animals need to tolerate each time is considerably larger than that of in vitro maxillae and rats cannot endure a 10-day radiation dose. Therefore, the method of measuring bone volume for the same-sized ROI in rats was used in this study to illustrate the effect of SrR on bone formation in the mid-palatal suture.

BMP-2 is a key member of the BMP family, and is known to promote formation of cartilage and bone. BMP-2 can induce differentiation of mesenchymal stem cells into osteoblasts and promote proliferation and differentiation of osteoblasts and their precursor cells to enhance cartilage and bone formation. ${ }^{51,52}$ Additionally, BMP-2 can stimulate the synthesis and secretion of osteoprotegerin, which inhibits the differentiation and maturation of osteoclasts. ${ }^{53}$ Recent studies $^{54}$ show that SrR can induce osteoblast differentiation of rodent bone mesenchymal stem cells, and enhance alkaline phosphatase activity and formation of mineralized nodules through the BMP-2/Smad pathway. Our results show that BMP-2 expression was higher in the SE group than in the EO group at each time point during the study. BMP-2 has been reported to induce bone formation by intramembranous ossification. ${ }^{55}$ Therefore, the observation that SrR stimulated BMP-2 indirectly supports a stimulatory effect of SrR on bone formation in the expanded midpalatal suture.

In conclusion, these results indicate that $\mathrm{SrR}$ can increase bone formation and may be effective in preventing relapse after RME. The study was designed to evaluate the effects of $\mathrm{SrR}$ on bone formation in the mid-palatal suture, so histological analyses of the drug were limited to the rodent mid-palatal suture. SrR might also have some influence on remodeling of the alveolar bone in the molars after RME. Moreover, experiments have only been done in animal models and we have not yet obtained evidence in humans, so we 
are a long way off implementing these findings in the clinic. Meanwhile, the precise mechanism via which SrR induces bone regeneration is still unclear. Therefore, further studies on SrR and bone remodeling should be designed.

\section{Conclusion}

SrR has positive effects on bone formation in the mid-palatal suture after RME in rats, and may be therapeutically beneficial in prevention of relapse and shortening of the retention period after RME.

\section{Acknowledgments}

The study was supported by the Science and Technology Development Program of Shandong Province (2014GGH218024) and the National Science Foundation of China (81371180).

\section{Author contributions}

All authors contributed toward data analysis, drafting and revising the paper and agree to be accountable for all aspects of the work.

\section{Disclosure}

The authors report no conflicts of interest in this work.

\section{References}

1. Saito S, Shimizu N. Stimulatory effects of low-power laser irradiation on bone regeneration in midpalatal suture during expansion in the rat. Am J Orthod Dentofacial Orthop. 1997;111(5):525-532.

2. Tang GH, Xu J, Chen RJ, Qian YF, Shen G. Lithium delivery enhances bone growth during midpalatal expansion. $J$ Dent Res. 2011;90(3):336-340.

3. Sawada M, Shimizu N. Stimulation of bone formation in the expanding mid-palatal suture by transforming growth factor-beta 1 in the rat. Eur J Orthod. 1996;18(2):169-179.

4. Barber AF, Sims MR. Rapid maxillary expansion and external root resorption in man: a scanning electron microscope study. Am J Orthod. 1981; 79(6):630-652.

5. Linder-Aronson S, Lindgren J. The skeletal and dental effects of rapid maxillary expansion. Br J Orthod. 1979;6(1):25-29.

6. Uysal T, Amasyali M, Enhos S, Sonmez MF, Sagdic D. Effect of ED-71, a new active vitamin $\mathrm{D}$ analog, on bone formation in an orthopedically expanded suture in rats. A histomorphometric study. Eur J Dent. 2009; 3(3):165-172.

7. Palma JC, Tejedor-Sanz N, Oteo MD, Alarcon JA. Long-term stability of rapid maxillary expansion combined with chincup protraction followed by fixed appliances. Angle Orthod. 2015;85(2):270-277.

8. Gurel HG, Memili B, Erkan M, Sukurica Y. Long-term effects of rapid maxillary expansion followed by fixed appliances. Angle Orthod. 2010;80(1):5-9.

9. McNamara JA Jr, Baccetti T, Franchi L, Herberger TA. Rapid maxillary expansion followed by fixed appliances: a long-term evaluation of changes in arch dimensions. Angle Orthod. 2003;73(4):344-353.

10. Gurgel JA, Tiago CM, Normando D. Transverse changes after surgically assisted rapid palatal expansion. Int J Oral Maxillofac Surg. 2014; 43(3):316-322.

11. Prado GP, Pereira MD, Bilo JP, Furtado F, Ferreira LM. Stability of surgically assisted rapid palatal expansion: a randomized trial. J Dent Res. 2013;92(7 Suppl):49S-54S.
12. Kilic E, Kilic B, Kurt G, Sakin C, Alkan A. Effects of surgically assisted rapid palatal expansion with and without pterygomaxillary disjunction on dental and skeletal structures: a retrospective review. Oral Surg Oral Med Oral Pathol Oral Radiol. 2013;115(2):167-174.

13. Chamberland S. Long-term dental and skeletal changes following surgically assisted rapid palatal expansion. Oral Surg Oral Med Oral Pathol Oral Radiol. 2013;116(1):120-121.

14. Goddard R, Witherow H. Surgically assisted rapid palatal expansion (SARPE). Br J Oral Maxillofac Surg. 2011;49(1):65-66.

15. Suri L, Taneja P. Surgically assisted rapid palatal expansion: a literature review. Am J Orthod Dentofacial Orthop. 2008;133(2):290-302.

16. Angeletti P, Pereira MD, Gomes HC, Hino CT, Ferreira LM. Effect of low-level laser therapy (GaAlAs) on bone regeneration in midpalatal anterior suture after surgically assisted rapid maxillary expansion. Oral Surg Oral Med Oral Pathol Oral Radiol Endod. 2010;109(3):e38-e46.

17. Uysal T, Amasyali M, Olmez H, Enhos S, Karslioglu Y, Gunhan O. Effect of vitamin $\mathrm{C}$ on bone formation in the expanded inter-premaxillary suture. Early bone changes. J Orofac Orthop. 2011;72(4):290-300.

18. Ozturk F, Babacan H, Inan S, Gumus C. Effects of bisphosphonates on sutural bone formation and relapse: a histologic and immunohistochemical study. Am J Orthod Dentofacial Orthop. 2011;140(1):e31-e41.

19. Uysal T, Ustdal A, Sonmez MF, Ozturk F. Stimulation of bone formation by dietary boron in an orthopedically expanded suture in rabbits. Angle Orthod. 2009;79(5):984-990.

20. Ozdemir H, Kara MI, Erciyas K, Ozer H, Ay S. Preventive effects of thymoquinone in a rat periodontitis model: a morphometric and histopathological study. J Periodontal Res. 2012;47(1):74-80.

21. Kara MI, Erciyas K, Altan AB, Ozkut M, Ay S, Inan S. Thymoquinone accelerates new bone formation in the rapid maxillary expansion procedure. Arch Oral Biol. 2012;57(4):357-363.

22. Marie PJ. Optimizing bone metabolism in osteoporosis: insight into the pharmacologic profile of strontium ranelate. Osteoporos Int. 2003;14 Suppl 3:S9-S12.

23. Bain SD, Jerome C, Shen V, Dupin-Roger I, Ammann P. Strontium ranelate improves bone strength in ovariectomized rat by positively influencing bone resistance determinants. Osteoporos Int. 2009;20(8):1417-1428.

24. Li YF, Luo E, Feng G, Zhu SS, Li JH, Hu J. Systemic treatment with strontium ranelate promotes tibial fracture healing in ovariectomized rats. Osteoporos Int. 2010;21(11):1889-1897.

25. Loddi PP, Pereira MD, Wolosker AB, Hino CT, Kreniski TM, Ferreira LM. Transverse effects after surgically assisted rapid maxillary expansion in the midpalatal suture using computed tomography. J Craniofac Surg. 2008;19(2):433-438.

26. Sannomiya EK, Macedo MM, Siqueira DF, Goldenberg FC, Bommarito S. Evaluation of optical density of the midpalatal suture 3 months after surgically assisted rapid maxillary expansion. Dentomaxillofac Radiol. 2007;36(2):97-101.

27. Betts NJ, Vanarsdall RL, Barber HD, Higgins-Barber K, Fonseca RJ. Diagnosis and treatment of transverse maxillary deficiency. Int $J$ Adult Orthodon Orthognath Surg. 1995;10(2):75-96.

28. Zemann W, Schanbacher M, Feichtinger M, Linecker A, Karcher H. Dentoalveolar changes after surgically assisted maxillary expansion: a three-dimensional evaluation. Oral Surg Oral Med Oral Pathol Oral Radiol Endod. 2009;107(1):36-42.

29. Halazonetis DJ, Katsavrias E, Spyropoulos MN. Changes in cheek pressure following rapid maxillary expansion. Eur J Orthod. 1994;16(4): 295-300.

30. Ozturk F, Babacan H, Gumus C. Effects of zoledronic acid on sutural bone formation: a computed tomography study. Eur J Orthod. 2012;34(2):141-146.

31. Meunier PJ, Roux C, Ortolani S, et al. Effects of long-term strontium ranelate treatment on vertebral fracture risk in postmenopausal women with osteoporosis. Osteoporos Int. 2009;20(10):1663-1673.

32. Meunier PJ, Roux C, Seeman E, et al. The effects of strontium ranelate on the risk of vertebral fracture in women with postmenopausal osteoporosis. $N$ Engl J Med. 2004;350(5):459-468. 
33. Hurtel-Lemaire AS, Mentaverri R, Caudrillier A, et al. The calciumsensing receptor is involved in strontium ranelate-induced osteoclast apoptosis new insights into the associated signaling pathways. $J$ Biol Chem. 2009;284(1):575-584.

34. Brennan TC, Rybchyn MS, Green W, Atwa S, Conigrave AD, Mason RS. Osteoblasts play key roles in the mechanisms of action of strontium ranelate. Br J Pharmacol. 2009;157(7):1291-1300.

35. Atkins GJ, Welldon KJ, Halbout P, Findlay DM. Strontium ranelate treatment of human primary osteoblasts promotes an osteocyte-like phenotype while eliciting an osteoprotegerin response. Osteoporos Int. 2009;20(4):653-664.

36. Choudhary S, Halbout P, Alander C, Raisz L, Pilbeam C. Strontium ranelate promotes osteoblastic differentiation and mineralization of murine bone marrow stromal cells: Involvement of prostaglandins. J Bone Miner Res. 2007;22(7):1002-1010.

37. Canalis E, Hott M, Deloffre P, Tsouderos Y, Marie PJ. The divalent strontium salt S12911 enhances bone cell replication and bone formation in vitro. Bone. 1996;18(6):517-523.

38. Takahashi N, Sasaki T, Tsouderos Y, Suda T. S 12911-2 inhibits osteoclastic bone resorption in vitro. J Bone Miner Res. 2003;18(6): 1082-1087.

39. Marie PJ, Hott M, Modrowski D, et al. An uncoupling agent containing strontium prevents bone loss by depressing bone resorption and maintaining bone formation in estrogen-deficient rats. J Bone Miner Res. 1993;8(5):607-615.

40. Latham RA. The development, structure and growth pattern of the human mid-palatal suture. J Anat. 1971;108 Pt 1:31-41.

41. Kanekawa M, Shimizu N. Age-related changes on bone regeneration in midpalatal suture during maxillary expansion in the rat. Am J Orthod Dentofacial Orthop. 1998;114(6):646-653.

42. Haas AJ. Long-term post-treatment evaluation of rapid palatal expansion. Angle Orthod. 1980;50(3):189-217.

43. Wertz RA. Skeletal and dental changes accompanying rapid midpalatal suture opening. Am J Orthod. 1970;58(1):41-66.

44. Strauss PG, Closs EI, Schmidt J, Erfle V. Gene expression during osteogenic differentiation in mandibular condyles in vitro. J Cell Biol. 1990;110(4):1369-1378.
45. Silbermann M, Reddi AH, Hand AR, Leapman RD, Von der Mark K, Franzen A. Further characterisation of the extracellular matrix in the mandibular condyle in neonatal mice. J Anat. 1987;151:169-188.

46. Hall BK. Selective proliferation and accumulation of chondroprogenitor cells as the mode of action of biomechanical factors during secondary chondrogenesis. Teratology. 1979;20(1):81-91.

47. Hanson NA, Bagi CM. Alternative approach to assessment of bone quality using micro-computed tomography. Bone. 2004;35(1):326-333.

48. Takenouchi H, Mayahara K, Arai Y, Karasawa Y, Shimizu N. Longitudinal quantitative evaluation of the mid-palatal suture after rapid expansion using in vivo micro-CT. Arch Oral Biol. 2014;59(4):414-423.

49. Hou B, Fukai N, Olsen BR. Mechanical force-induced midpalatal suture remodeling in mice. Bone. 2007;40(6):1483-1493.

50. Brun LR, Galich AM, Vega E, et al. Strontium ranelate effect on bone mineral density is modified by previous bisphosphonate treatment. SpringerPlus. 2014;3:676.

51. Matsubara T, Kida K, Yamaguchi A, et al. BMP2 regulates osterix through Msx 2 and Runx2 during osteoblast differentiation. J Biol Chem. 2008;283(43):29119-29125.

52. Li Z, Hassan MQ, Volinia S, et al. A microRNA signature for a BMP2induced osteoblast lineage commitment program. Proc Natl Acad Sci US A. 2008;105(37):13906-13911.

53. Song SJ, Jeon O, Yang HS, Ran DK, Kim BS. Effects of culture conditions on osteogenic differentiation in human mesenchymal stem cells. J Microbiol Biotechnol. 2007;17(7):1113-1119.

54. Lv H, Huang X, Jin S, Guo R, Wu W. [Strontium ranelate promotes osteogenic differentiation of rat bone mesenchymal stem cells through bone morphogenetic protein-2/Smad signaling pathway]. Nan Fang Yi Ke Da Хие Xие Bao. 2013;33(3):376-381. Chinese.

55. Stoeger T, Proetzel G, Welzel H, et al. In situ gene expression analysis during BMP2-induced ectopic bone formation in mice shows simultaneous endochondral and intramembranous ossification. Growth Factors. 2002;20(4):197-210.
Drug Design, Development and Therapy

\section{Publish your work in this journal}

Drug Design, Development and Therapy is an international, peerreviewed open-access journal that spans the spectrum of drug design and development through to clinical applications. Clinical outcomes, patient safety, and programs for the development and effective, safe, and sustained use of medicines are a feature of the journal, which

\section{Dovepress}

has also been accepted for indexing on PubMed Central. The manuscript management system is completely online and includes a very quick and fair peer-review system, which is all easy to use. Visit http://www.dovepress.com/testimonials.php to read real quotes from published authors. 